Journal of

Radiotherapy

in Practice
Fournal of Radiotherapy in Practice (2008)

7, 59-59

(C) 2008 Cambridge University Press

doi: $10.1017 /$ S1460396908006286

\title{
Letter
}

\section{GTV and CTV in lung cancer}

Ignacio Alastuey

Clinical target volume (CTV) expresses the concept of gross tumour volume (GTV) plus a margin accounting for subclinical disease. This is made by adding a volume of normal lung tissue around GTV, not really knowing if this volume includes malignant cells or not.

If this volume contains no malignant cells, then in that case normal lung tissue will receive the prescribed dose to planning target volume (PTV) (CTV plus margins). Normal tissue complication probability is related to dose to total lung normal tissue (both lungs) expressed in concepts such as v20. If total normal lung tissue is reduced because the patient gets smaller, there will be an increased v20 compared to v20 with smaller CTV /total lung volume (bigger patients).

This might be pointing to the need of decreasing CTV margins as the total lung volume is decreased, or in an extreme to make CTV = GTV in lung cancer, preserving the concept of PTV.

Ignacio Alastuey

Radiotherapy Hospital San Dureta

Andrea Duria 5507019

Palma de Mallorca

Spain

Email: repulsivo@yahoo.es 